\title{
Surgical Intervention in Sub-Arachnoid Cyst with Severe Neurological Manifestations
}

\author{
Dinyadarshini Johnson ${ }^{1}$, Chieng Xing Yee ${ }^{2}$, Sharon Casilda Theophilus ${ }^{3}$ and Anil Gandhi ${ }^{4 \star}$ \\ ${ }^{\prime}$ Medical Graduate, Monash University, Malaysia \\ ${ }^{2}$ Kuching General Hospital, Malaysia \\ ${ }^{3}$ Consultant Neuro-Surgeon, Hospital Sultanah Aminah, Malaysia \\ ${ }^{4}$ Associate Professor, Monash University, Malaysia
}

\section{Article Info}

\author{
*Corresponding author: \\ Anil Gandhi \\ Associate Professor \\ Monash University Malaysia Sdn Bhd, \\ Jalan Lagoon Seiatan \\ Bandar Sunway \\ 47500 Petaling Jaya Selangor \\ Malaysia \\ E-mail: anil.gandhi@monash.edu
}

Received: November 11, 2017

Accepted: February 11, 2018

Published: February 16, 2018

Citation: Johnson D, Yee CX, Theophilus SC, Gandhi A. Surgical Intervention in SubArachnoid Cyst with Severe Neurological Manifestations. Madridge J Neurosci. 2018; 2(1): 47-50.

doi: $10.18689 /$ mjns- 1000108

Copyright: ๑ 2018 The Author(s). This work is licensed under a Creative Commons Attribution 4.0 International License, which permits unrestricted use, distribution, and reproduction in any medium, provided the original work is properly cited.

Published by Madridge Publishers

\begin{abstract}
This case looks at a 25 year old Chinese female with left cerebellar arachnoid cyst who has gone under sub-occipital craniotomy and marsupialisation of the cyst. The patient was clinically symptomatic, which prompted surgical intervention. Clinical assessments were done a day prior to surgery and subsequently on day 3 post-surgery. Our discussions are based upon the current literature review on conservative versus surgical management of a patient with arachnoid cyst. The discussion also further examines the clinical advantage of marsupialisation.
\end{abstract}

Keywords: Surgical Intervention; Sub-Arachnoid Cyst; Surgical management; Marsupialisation.

\section{Introduction}

Arachnoid cysts are cerebrospinal fluid covered by arachnoidal cells and collagen that may develop on the arachnoid membrane on intracranial or on spinal cord [1]. It is a rare condition which is usually seen in paediatric population [3]. The aetiology can be primary or secondary in origin if the exact mechanism is yet to be definitely established. Primary arachnoid cysts occur congenitally due to the abnormal development of the brain and spinal cord whereas secondary arachnoid cysts may develop as a result of a head trauma, meningitis, malignancy, metastatic lesions, or brain surgery [1]. There are theories that suggest the cysts occur as a result of developmental malformations. However, this becomes secondary as the tearing of the arachnoid membrane is still unknown, along with its enlargement over time in juxtaposition to the ball valve effect $[2,3]$. There are also familial patterns observed in various cases that could suggest a genetic predisposition that contributes to the occurrence of arachnoid cyst in an individual $[2,4]$. Although by and large, it remains asymptomatic and the clinical symptoms may manifest during infancy or early adulthood [1]. The symptoms, however, may vary according to the location and size of the cyst. The typical clinical manifestations may include headache, nausea, vomiting, seizure, visual and hearing disturbances, unsteady gait with notable lack of balance, and coordination suggesting cerebellar involvement $[1,2]$. Other findings include hemiparesis, and rarely, increased intracranial pressure as a manifestation of hydrocephalus [2]. The diagnosis is often made incidentally, especially in someone presenting with seizure based on a Computerized Tomography (CT) scan or Magnetic Resonance Imaging (MRI) of the brain [2]. The clinical interventions are necessitated based on the severity of the clinical symptoms. Most arachnoid cysts are asymptomatic and do not require treatment, however, treatment may be initiated when patients are symptomatic. There are various treatment modalities available to treat sub arachnoid cysts depending on the location and size of the cyst. For example, if the cyst is small and not causing discernible symptoms, it is advisable 
not to treat them. If the cyst is large and causing glaring symptoms, there are various treatment options available contingent upon the size and severity of symptoms.

There are several treatment options available to decompress the cysts. For example, a placement of cerebral shunt or internal "shunt" would drain into the subdural compartment. Another option would be a cystoperitoneal shunt that would drain into the peritoneal cavity opr simply by needle aspiration. Additionally, another option is craniotomy with excision of the cyst and various endoscopic techniques have also been proven effective in decompressing the shunt. Pharmacological treatment may be required to deal with some severe symptoms like seizures or pain.

\section{Case Report}

\section{History}

This case looks at a 25 year old Chinese female who works as a logistics coordinator. She has no known medical illness and was screened after two days of vomiting, there months prior to surgical intervention. She presented with frequent vomiting often post-meal consumption, therefore containing food particles. She also complained of throbbing headache in the left occipital region for a couple of months prior to recurring vomiting. She was initially diagnosed as having "food poisoning" and treated over a day admission in the general hospital. However, her symptoms persisted, prompting further investigation.

CT brain revealed some "abnormality" in her brain, indicating neurosurgery referral and MRI for further evaluation. After a few months, the patient then had her subsequent follow-up in a tertiary referral hospital under the neurosurgery department. During this interval, the patient reported having persistent vomiting, noticeable weight loss, throbbing headache, and unsteady gait as the patient claims she was unable to maintain balance with tendency to sway to the left and double vision. These symptoms, in addition to lethargy, interfered with her routine activities. Further reviews of other systems were all unremarkable.

Her past history revealed that she was involved in a motor vehicle accident when she was 6 years old and sustained mild injuries on her forehead, with no loss of consciousness, requiring neither hospital admission nor major surgical intervention.

\section{Physical Examination}

The patient was alert and conscious during admission and her cognitive functions were intact. She is right handed. Cranial nerve examination reported diplopia with right upward gaze and nystagmus of the right eye. Her motor and sensation were intact, except for brisk reflexes in the lower limb. Finger-nose test and heel-shin test were noted positive on the left side. She demonstrated an unsteady, wide-based gait with a tendency to sway towards the left side. Romberg test was positive.

\section{Investigations}

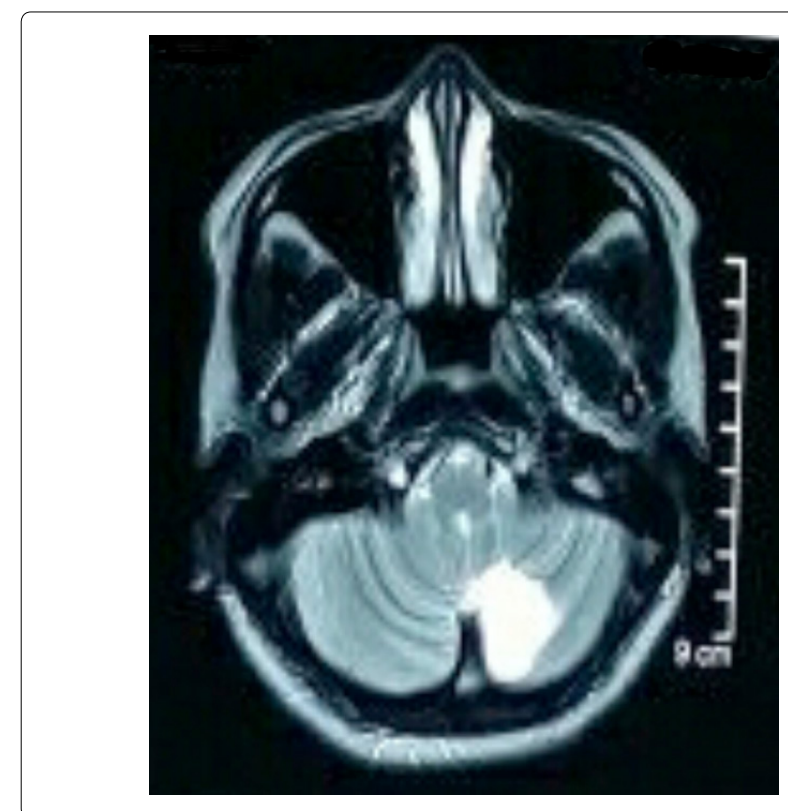

Figure 1. MRI image T2 weighted showed a hyper-intense area at left cerebellar at T2 area, measuring $2 \mathrm{~cm}$ by $1.5 \mathrm{~cm}$ in size was suggestive of cystic lesion.

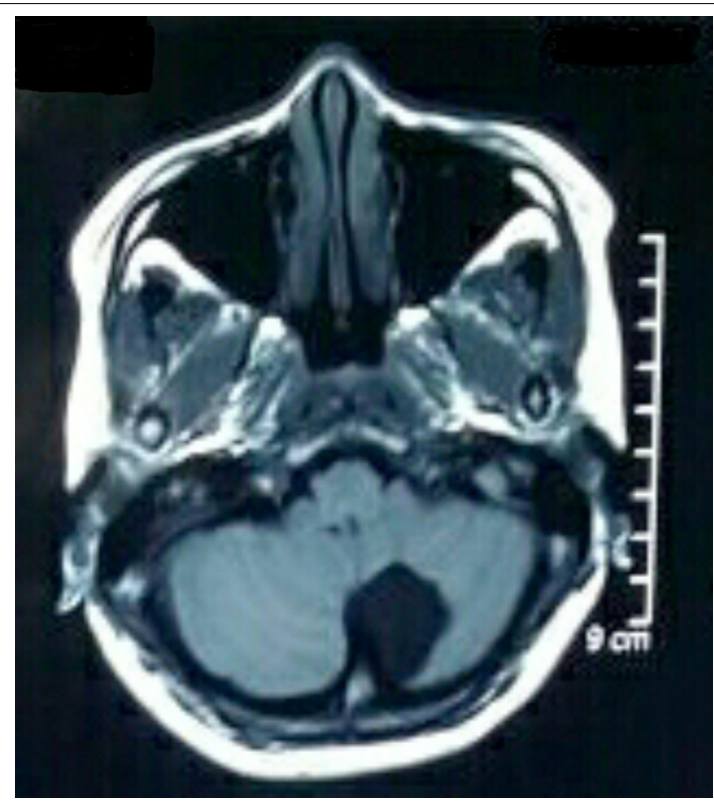

Figure 2. MRI T1 weighted with contrast with an enhanced hypointense lesion at left cerebellar.

\section{Surgical Management}

Considering her symptoms and MRI findings, surgical intervention was considered to the best option. The patient underwent Sub occipital craniotomy and marsupialization of arachnoid cyst. There were no surgical complications.

\section{Post-Surgical Clinical Assessment}

Patient recovered well and was discharged on day 4 postsurgery. Clinical assessment post-surgery revealed remarkable improvements in terms of clinical signs and symptoms exhibited by the patient prior to surgical intervention. Upon discharge, patient was able to walk steadily with resolved symptoms of vomiting, headache, and double vision. 


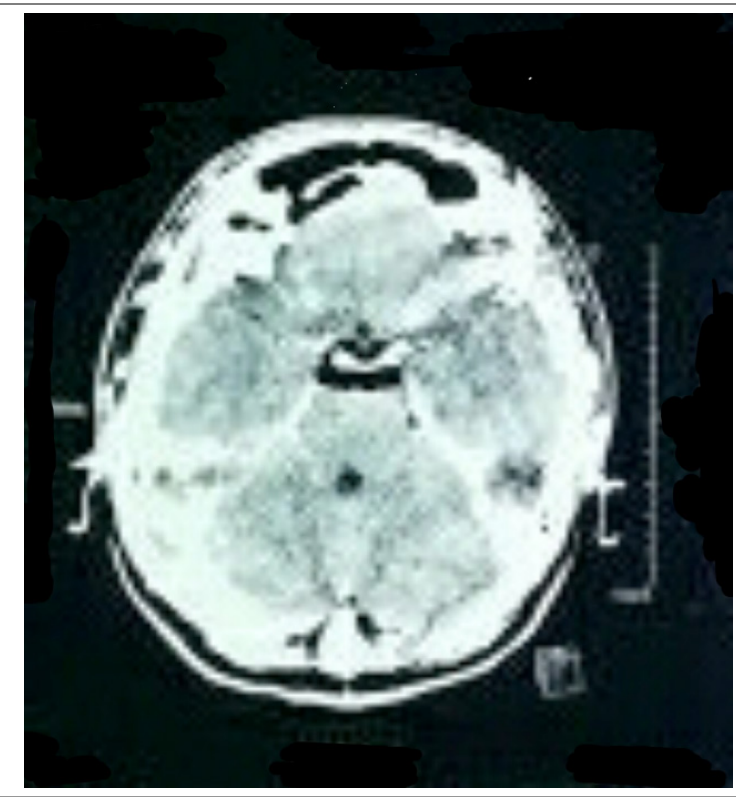

Figure 3. CT scan Image taken postoperatively showing no cystic lesion.

\section{Discussion \\ Conservative Versus Surgical Management In A Patient With Arachnoid Cyst}

In general, arachnoid cyst are usually conservatively managed, however in this case, given the compressive effect of the brain lesion, neurological symptoms formed. These began to affect her daily activities and treating with occipital craniotomy and marsupialization of the cystic lesion was advised. The cyst wall was removed, except the part that was adhered to the cerebrum vascular structures. Subsequent CT scan of the brain showed postoperative changes and pneumocranium after the removal of the left cerebellar arachnoid cyst.

To date, the best treatment option for arachnoid cysts remains controversial. For asymptomatic patients with arachnoid cysts, the current and widely adopted practice is conservative management [7]. The reason being surgical intervention has high morbidity and the size of most cysts remain unchanged. These patients are monitored closely with CT and MRI scanning $[6,7]$. Surgery is recommended for patients who exhibited signs of raised intracranial pressure secondary to the arachnoid cyst, focal neurological signs, or complications such as subdural hematoma, hydrocephalus or in specific patients with epilepsy which can affect patient's daily living [8]. However, some authors believe that mass effect produced by the arachnoid cyst inadvertently pose as a risk and may lead to subsequent complications to asymptomatic patients and this may warrant surgical intervention $[7,8]$. Therefore, the treatment chosen is highly individualized. In addition, none of the studies reviewed reported mortality associated with the surgical management of the arachnoid cysts $[5,7,9,10]$.

\section{Marsupialization Versus Cystoperitoneal Shunt In Treating Arachnoid Cyst}

The best surgical modality for the treatment of arachnoid cyst still remains controversial to date [5]. Several studies, based on case reviews, have explored various surgical modality in proportion to the characteristic and location of the cyst, clinical manifestations, technical viability, and outcome of the procedure $[5,7,9]$. In a retrospective review of 32 cases of arachnoid cyst of which $75 \%$ were managed surgically in a single institution from 1994-2005, revealed that excision and marsupialization was a common procedure performed ensuring positive clinical outcomes and no deterioration. $64 \%$ of the cases were reported to have reduction in the size of the cysts, $18 \%$ with complete resolution of the cysts, and no changes noted in the remaining cases [5]. Overall, there was recurrence rate of $29 \%$ in cases that were managed surgically with excision and marsupialization accounting for the majority.

In another study, which reviewed 209 cases of symptomatic arachnoid cysts treated from 1986-2008 using various surgical modalities including endoscopic fenestration, shunt placement and marsupialization showed that cystoperitoneal shunt was the best modality for a rapid obliteration of the cyst and improved radiological outcome [9]. Although this method promises rapid improvement, there are significant drawbacks in terms of shunt dependencies and malfunctions, infection and unexpected hemorrhage $[7,9]$. The technical viability still remain debatable and difficulty may arise in small-sized cysts and cysts located in the posterior fossa and suprasellar. It was also highlighted that open surgery marsupialization is as effective as shunting in obliteration of the cysts with an added advantage of being shunt-independent on a note that major surgical-related complications, including subdural hematoma, meningitis, neurological deficit, and even death in the former [9]. Limited studies are available for the treatment of arachnoid cysts in the posterior fossa due to its location, therefore, the best surgical approach is still yet to be clearly established [9, 10]. In a retrospective analysis, 12 patients with arachnoid cyst in the posterior fossa were subjected to open surgery for complete resection of the cyst. Remarkable improvements in one of the reported clinical systems were noted immediately after surgery. This method was also deemed most likely to prevent recurrence of the cysts and showed improvements over a long-term follow-up. In addition, other surgical modalities, including shunting, appeared to be hazardous in the treatment of posterior fossa arachnoid cyst due to its close relation to vital neurovascular structures. In these cases, suboccipital approach promises a safe and effective surgical outcome. On the other hand, cystoperitoneal shunt was recommended as the first choice for the management of arachnoid cyst in the posterior fossa with hydrocephalus as it provided good clinical outcomes with low recurrence rate [10].

In our case, the patient had no clinical (no papilloedema) or radiological evidence of hydrocephalus. The patient saw considerable improvements by day 4 post surgery, with most of her symptoms completely resolved. Marsupialization of the arachnoid cyst in the posterior fossa appeared to be the best surgical modality due to the location and size of the cyst. Cystoperitoneal shunt was not the preferred choice of treatment given the small-sized cyst in the cerebellum, with inevitable difficulty in the technical viability of the shunt. 


\section{Conclusion}

Based on our discussion, surgical intervention in symptomatic patients ensued good clinical outcomes. However, the surgical modality is best-suited to the patient, with consideration given to various aspects involving the cyst and technical viability of the proposed surgery. Often, decision is made collectively by incorporating existing scientific evidence, availability of resources, and the neurosurgeon's experience. The choice of surgical treatment is often individualised and largely dependent on the clinical manifestations, characteristic, location of the cyst, technical viability, availability of the resources, and the competence of the operating neurosurgeon. Open surgery still remains a common and accepted procedure by most of the neurosurgeons, although with technological advancement and more trained surgeons, endoscopic management may eventually overtake.

\section{References}

1. Arachnoid Cysts Information Page: National Institute of Neurological Disorders and Stroke (NINDS). Ninds.nih.gov. 2016.

2. Arachnoid Cysts - NORD (National Organization for Rare Disorders). NORD (National Organization for Rare Disorders). 2016.

3. Norden A, Chheda M, Wen P. Uncommon brain tumors. 2016 [cited 2 October 2016].

4. Khan A, Macdonald S, Mahmood K, Turnbull I, Al-Okaili R, et al. Arachnoid Cyst Imaging: Overview, Radiography, Computed Tomography. 2016.

5. Boutarbouch M, El Ouahabi A, Rifi L, Arkha Y, Derraz S, et al. Management of intracranial arachnoid cysts: Institutional experience with initial 32 cases and review of the literature. Clinical Neurology and Neurosurgery. 2008; 110(1): 1-7. doi: 10.1016/j.clineuro.2007.08.009

6. Pradilla G, Jallo $G$. Arachnoid cysts: case series and review of the literature. Int J Gastrointest Cancer. 2007; 22(2): 7.

7. Cincu R, Agrawal A, Eiras J. Intracranial arachnoid cysts: Current concepts and treatment alternatives. Clinical Neurology and Neurosurgery. 2007; 109(10): 837-843. doi: 10.1016/j.clineuro.2007.07.013

8. Gosalakkal J. Intracranial arachnoid cysts in children: a review of pathogenesis, clinical features, and management. Pediatric Neurology. 2002; 26(2): 93-98. doi: 10.1016/S0887-8994(01)00329-0

9. Shim KW, Lee YH, Park EK, Park YS, Choi JU, et al. Treatment options for arachnoid cysts. Childs Nerv Syst. 2009; 25(11): 1459-1466. doi: 10.1007/ s00381-009-0930-7

10. Samil M, Carvalho GA, Schuhmann MU, Matthies C. Arachnoid cysts of the posterior fossa. Surg Neurol. 1999; 51(4): 376-82. 